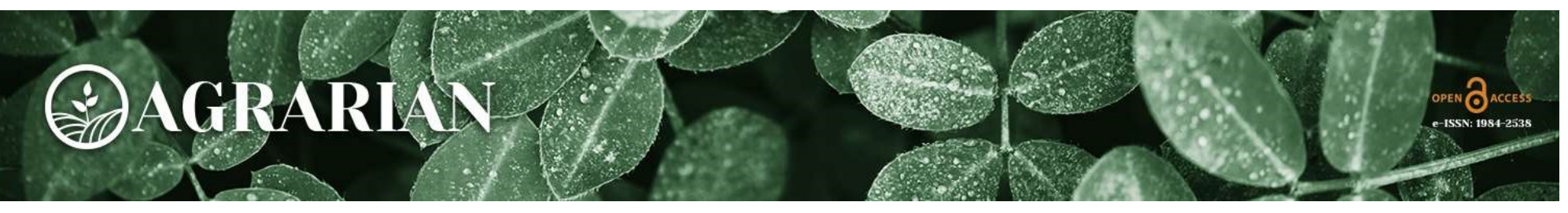

\title{
Comprimento do talhão na operação de preparo do solo com grade média
}

\author{
Field length in the soil preparation operation with medium harrow
}

\author{
Antonio Alves Pinto \\ Universidade Estadual Paulista \\ E-mail: antonioalvesunesp@gmail.com \\ OrclD: https://orcid.org/0000-0001-8615-2387 \\ Leandro Alves Pinto \\ Universidade Federal do Cariri \\ E-mail: leandroalvespinto96@gmail.com \\ OrclD:https://orcid.org/0000-0002-7132-6177 \\ Felipe Thomas da Camara \\ Universidade Federal do Cariri \\ E-mail: felipe.camara@ufca.edu.br \\ OrclD: https://orcid.org/0000-0003-2853-9908
}

\begin{abstract}
Resumo A utilização da mecanização agrícola tornou mais eficiente as práticas agrícolas, destacando-se o trator que permitiu a realização de práticas de cultivo mais eficientes. Portanto, objetivou-se determinar o comprimento de talhão que obtenha eficiência de campo de $90 \%$ na operação de preparo do solo com grade média em função da velocidade de deslocamento e do comprimento do talhão. O experimento foi realizado em delineamento inteiramente casualizado, em esquema fatorial $8 \times 3$, com seis repetições, totalizando 96 observações. O primeiro fator foram oito comprimentos de talhões $(30,60,90,120,150,180,210$ e $240 \mathrm{~m})$. O segundo foram três velocidades de deslocamento $\left(3,0 ; 4,1\right.$ e $\left.4,7 \mathrm{~km} \mathrm{~h}^{-1}\right)$. Com os dados de rendimento de campo efetivo foi gerado uma curva de resposta para seleção do comprimento de talhão que proporcione rendimento de campo efetivo de $90 \%$. No fator velocidade de deslocamento a maior capacidade de campo operacional ocorreu com $4,7 \mathrm{~km} \mathrm{~h}^{-1}$. Para o fator comprimento do talhão a maior capacidade de campo operacional e rendimento de campo efetivo foi observada com $240 \mathrm{~m}$. O rendimento de campo efetivo de $90 \%$ é alcançado com comprimento de talhão de 120 metros. A capacidade de campo máxima ocorreu com a velocidade de $4,7 \mathrm{~km} \mathrm{~h}^{-1}\left(0,93 \mathrm{ha} \mathrm{h}^{-1}\right)$ com o comprimento de talhão de $214 \mathrm{~m}$.
\end{abstract}

Palavras-chave: capacidade de campo, eficiência de campo, velocidade

Abstract: The use of agricultural mechanization has become more efficient in several agricultural practices, highlighting the tractor as the main machine used in carrying out these activities, thus allowing more efficient cultivation practices. The objective was to determine the field length that obtains field efficiency of $90 \%$ in the soil tillage operation with a medium harrow depending on the displacement speed and the length of the field. The experiment was carried out in a completely randomized design, in an $8 \times 3$ factorial scheme, with six replications, totaling 96 observations. The first factor was eight field lengths (30,60, 90, 120, 150, 180, 210 and $240 \mathrm{~m})$. The second was three travel speeds $(3.0 ; 4.1$ and $4.7 \mathrm{~km} \mathrm{~h}-1)$. With the effective field yield data, a response curve was

Agrarian, Dourados, v. 13, n. 49, p. 419-425, 2020. 


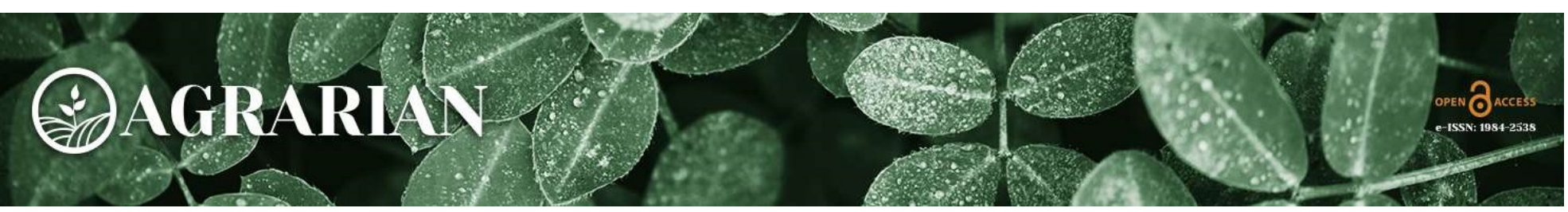

campo operacional foram observados com a velocidade de $4,7 \mathrm{~km} \mathrm{~h}^{-1}$. Tais resultados corroboram com Nagahama et al. (2013), ao observarem aumento da capacidade de campo operacional de 0,19 para 0,49 ha $\mathrm{h}^{-1}$, com acréscimo da velocidade de 2,45 para $6,5 \mathrm{~km} \mathrm{~h}^{-1}$. Tais resultados ocorrem devido ao fato de o aumento da velocidade reduzir o tempo gasto com preparo do solo.

Verifica-se na análise de regressão da interação na capacidade de campo operacional (Tabela 1), que todas as velocidades obtiveram significância $(p<0,01)$ para ambos os modelos de regressão. Nota-se que na capacidade de campo operacional para as três velocidades de trabalho o modelo polinomial de segunda ordem foi o mais representativo (Figura 1), sendo a máxima capacidade de campo operacional de 0, 54, 0, 74 e 0, $93 \mathrm{ha} \mathrm{h}^{-1} \mathrm{com}_{0}$ comprimento de talhão de 190, 193 e 214 m, respectivamente.

$\mathrm{Na}$ análise de regressão para o fator rendimento de campo efetivo (Tabela 1), verifica-se que ocorreu significância $(p<0,01)$. Conforme Ramos et al. (2016), quanto maior o comprimento dos talhões, melhor a eficiência de campo (RcE), sendo que se fosse possível não realizar manobras, o rendimento de campo efetivo seria $100 \%$. Porém, segundo Balastreire (1987), valores de $90 \%$ são considerados ótimos para operações de preparo de solo. Considerando o rendimento de campo efetivo de $90 \%$ e por meio da equação de regressão (Figura 2), obteve-se comprimento de talhão de 117,8 metros.

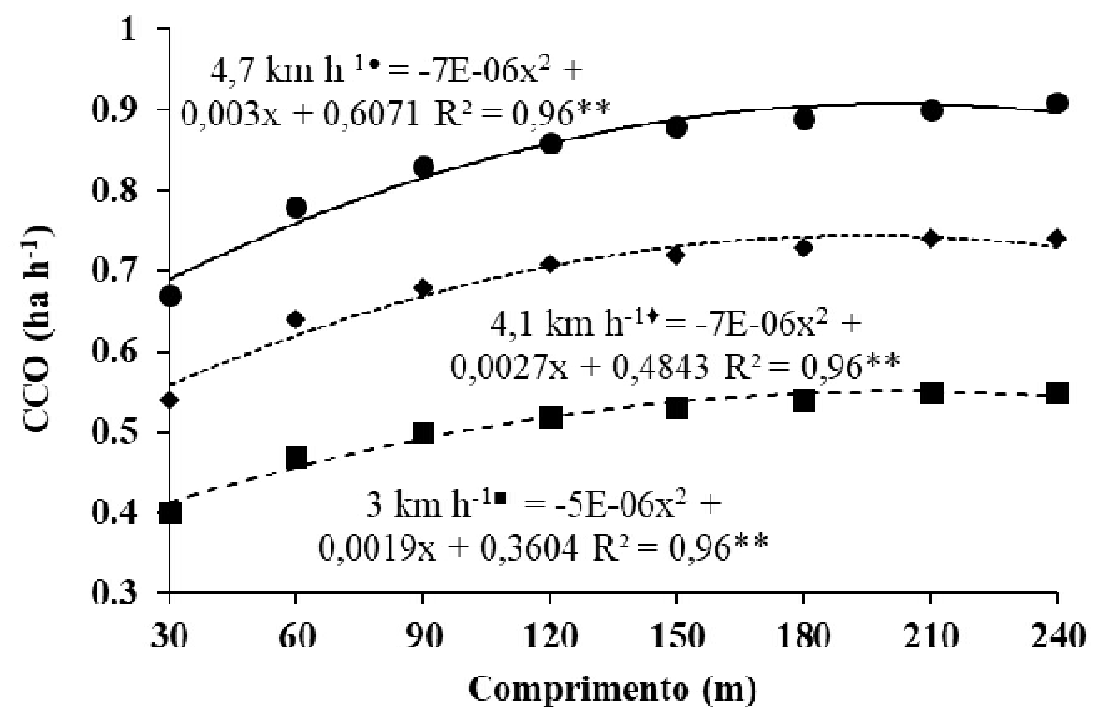

Figura 1. Análise de regressão para a capacidade campo operacional (CCO), nas três velocidades 

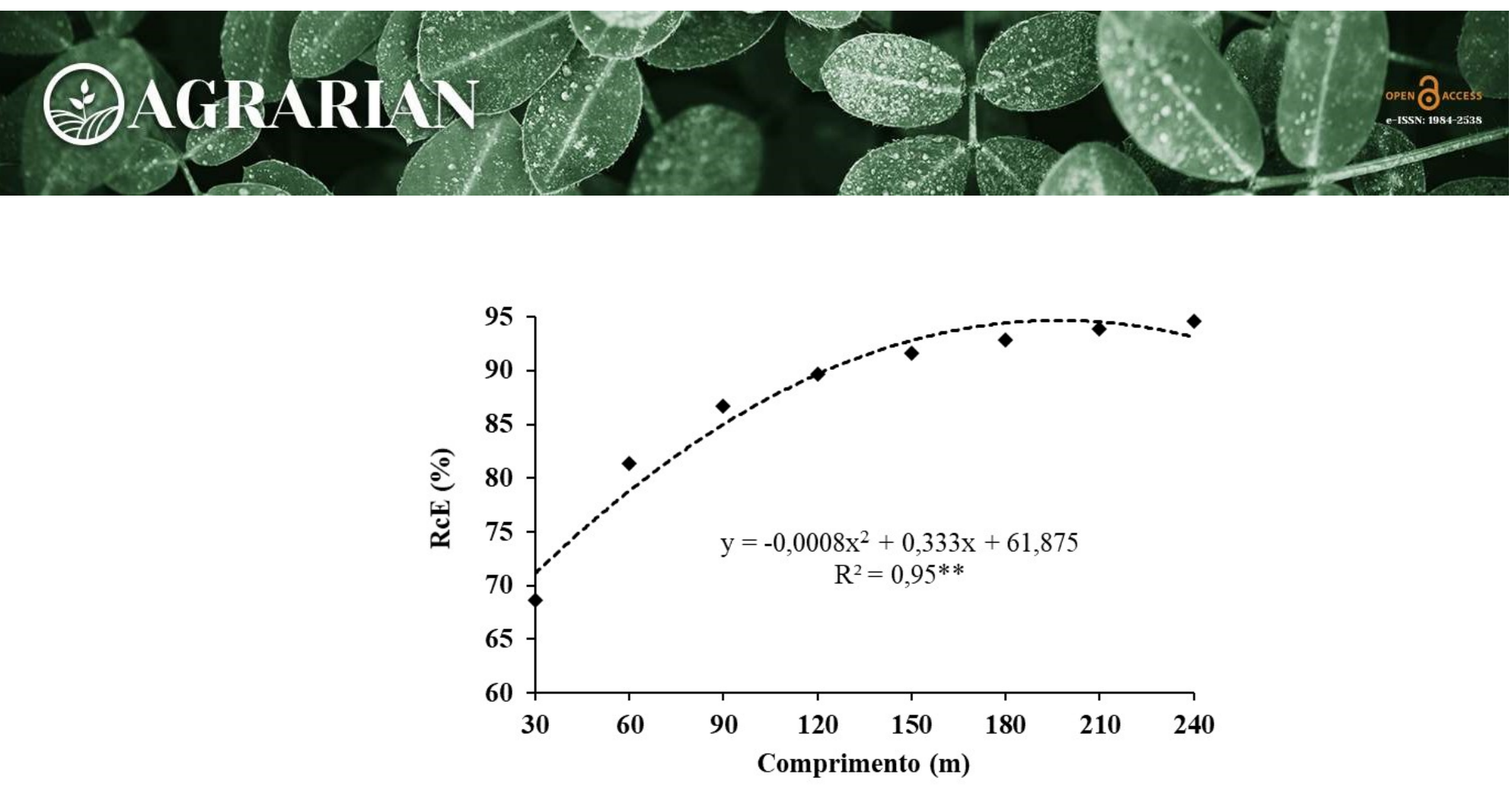

Figura 2. Análise de regressão para o rendimento de campo efetivo em função do comprimento do talhão

Nota-se na Figura 2, que a partir de $120 \mathrm{~m}$ de comprimento de talhão, os ganhos em rendimento de campo efetivo são pequenos, não justificando o uso de talhões maiores baseando-se apenas no ganho de rendimento na operação de preparo do solo com grade média.

\section{Conclusões}

O rendimento de campo efetivo de $90 \%$ é alcançado com comprimento de talhão de 120 metros $\mathrm{A}$ capacidade de campo operacional máxima ocorreu com a velocidade de $4,7 \mathrm{~km} \mathrm{~h}^{-1}\left(0,93 \mathrm{ha} \mathrm{h}^{-1}\right) \mathrm{com}^{\circ}$ comprimento de talhão de $214 \mathrm{~m}$.

\section{Referências}

ARAÚJO, V, F.; HONORATO, A. C.; CORTEZ, J. W.; PATROCÍNIO FILHO, A, P.; NAGAHAMA, H, J. Patinagem, opacidade e ruído de três tratores agrícolas de pneus. Revista Energia na Agricultura, v. 29, n.1, p. 01-08, 2014.

BALASTREIRE, L. A. Máquinas agrícolas. São Paulo: Manole, 1987. 307p

CORTEZ, J. W.; FERREIRA, B. J. M.; ALVES, A. D. S.; MOURA, M. R. D. NAGAHAMA, H. J. Desempenho operacional do conjunto trator-implementos nas operações de preparo do solo. Nucleus, v.8, n.2, p. 177-184, 2011.

CUNHA, J. P. B.; SILVA, F. M.; DIAS, R. E. B. A. Eficiência de campo em diferentes operações mecanizadas na cafeicultura. Coffee Science, v. 11, n. 1, p. 76-86, 2016.

FERNANDES, J.C.; GAMERO, C.A. Avaliação do desempenho das máquinas agrícolas na implantação da cultura do girassol. Energia na Agricultura, vol. 25, n.2, p.74-87, 2010.

MARTINS, M. B.; SANDI, J.; SOUZA, F. L.; SANTOS, R. S.; LANÇAS, K. P. Otimização energética de um trator agrícola utilizando normas técnicas em operações de gradagem. Revista Engenharia na Agricultura, V.26, n.01, p.52-57, 2018.

Agrarian, Dourados, v. 13, n. 49, p. 419-425, 2020. 
